\title{
The Effect of Dynamic Singularities on Robotic Control and Design
}

\author{
Bill Goodwine and Jason Nightingale
}

\begin{abstract}
This paper presents the definition of a new type of dynamic singularity for robotic manipulators. It is applicable to all underactuated robotic systems that can be described by Lagrange's equations where the Lagrangian is the kinetic minus potential energy. The approach is to decompose the velocity at every point in the configuration space into velocity directions that can be directly controlled and those that are uncontrolled and orthogonal to the directly controlled directions. These uncontrolled directions are controlled only through the dynamic coupling with the controlled directions and the measure of a dynamic singularity is then a measure of this degree of coupling. When this coupling is zero, the mechanism is said to be at a dynamic singularity. The practical implication is that, at such points, the dynamics are decoupled and control over the uncontrolled directions is very weak in that the mechanism will have to move away from the singularity before the inputs can affect the uncontrolled velocity directions. An example that is realistically complicated is presented and simulations show the effect on control inputs when the system is operating near a dynamic singularity.
\end{abstract}

\section{INTRODUCTION}

This paper presents a proposed metric for dynamic singularities for robotic mechanical systems. Specifically for an underactuated robotic system with equations of motion that can be described by Lagrange's equations, this metric provides a measure of the degree of coupling between the actuated and unactuated degrees of freedom for the system. Since the unactuated degrees of freedom can only be controlled through dynamic coupling with the directly controlled degrees of freedom, configurations where such coupling is zero are problematic if it is necessary to reject disturbances in the uncontrolled degrees of freedom.

This work extends the applicability of some previous results by the authors [10-12], which focused on the open problem in nonlinear control of determining conditions for controllability of mechanical systems starting from non-zero velocity. The prior work generally focused on nonholonomic mechanical systems such as the well-known snakeboard $[1,5,6,13]$ or the rollerblader [8]; whereas, the focus in this paper is directed more toward nonlinear manipulator-type mechanisms. However, it is emphasized that the approach is general and applicable to a very broad class of mechanical systems as is much of the work on control of nonlinear mechanical systems such as in [6, 7, 9]. As is nearly universally true in the field, the results in this paper are limited to nonlinear mechanical robotic systems which have

Bill Goodwine is with the Department of Aerospace \& Mechanical Engineering, University of Notre Dame, Notre Dame, Indiana 46556 USA goodwine@controls. ame.nd.edu

Jason Nightingale is with the Department of Aerospace \& Mechanical Engineering, University of Notre Dame, Notre Dame, Indiana 46556 USA jnightinend.edu

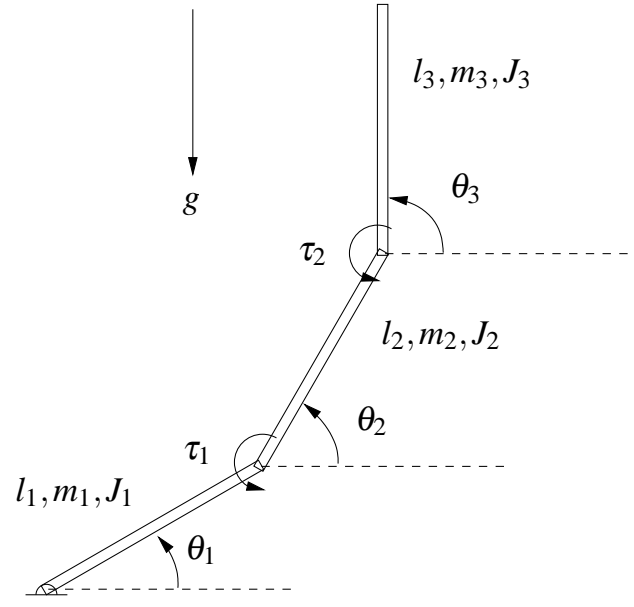

Fig. 1. Underactuated three-link manipulator with joints 2 and 3 actuated and joint 1 unactuated.

a Lagrangian that the difference between the kinetic and potential energies of the system.

In this paper we establish a framework for using the metric in mechanism design so that, if the disturbances that the system will normally be subjected to can be characterized, the system can be designed to either normally operate away from the dynamically singular configurations or optimize the location of the actuators for increased effectiveness. The main contributions of this paper are 1) a demonstration of the serious effects of the singularity; and 2) evaluation of the metric on a system with realistically-complicated dynamics (the underactuated triple-pendulum illustrated in Figure 1). Without the general formulation and the aid of a symbolic mathematics program, computing the metric and location of the singularities would be practically impossible for realistically-complicated robotic mechanisms.

This work refers to configurations in which there is complete decoupling between the controlled and uncontrolled velocity directions as dynamic singularities. This term has been used previously in slightly different ways that, while not identical to the present use, are similar to it in nature. For example, [15] considers dynamic singularities to be states of a robot that are impossible to attain for causes related to the robot's dynamics. Examples include configurations in which the moment of inertia about an axis goes to zero, which is physically impossible, but a common element of many modeling approaches. In [14], a dynamic singularity is a configuration in which a free-floating manipulator is unable 
to move its end-effector in some inertial direction.

Much like a kinematic singularity, our definition of a dynamic singularity is a function of the robot's configuration and parameters and not a function of its joint velocities. Unlike a kinematic singularity, however, it also depends on the system's inertial properties such as mass and moments of inertia. Just as a kinematic singularity can be both bad (necessitating large joint velocities) and good (large mechanical advantage), a dynamic singularity has both desirable and undesirable properties. The primary undesirable property is the aforementioned lack of control authority. A desirable property is that if the nature of the disturbances experienced by the mechanism near a dynamic singularity are generally aligned only with the controlled directions, the uncontrolled directions are isolated from those disturbances.

\section{Derivation of Dynamic Stability Metric}

This section derives the relevant terms for the dynamic singularity metric. A complete exposition on the basis for the description of mechanical systems used in this work can be found in $[2,4]$.

It is a standard result in mechanical systems theory that for a mechanical system with a Lagrangian $L=T-V$, a curve $\gamma(t)$ satisfies the Lagrange-d'Alembert principle if it satisfies

$$
\nabla_{\dot{\gamma}(t)} \dot{\gamma}(t)=\mathbb{G}^{\sharp}(F(t))-\operatorname{grad} V(\gamma(t)),
$$

where $\nabla$ denotes the covariant derivative and $\mathbb{G}^{\sharp}$ is standard notation for mechanical systems and, in matrix form, is simply the inverse of the inertia matrix. In coordinates, this may be expressed as

$$
\ddot{\theta}^{i}+\Gamma_{j k}^{i} \dot{\theta}^{j} \dot{\theta}^{k}=\tau^{a} \mathbb{G}^{i k} F_{k}^{a}-\mathbb{G}^{i k} \frac{\partial V}{\partial \theta_{k}},
$$

where superscripts on the inertia tensor indicate the usual tensor notation that they are the components of its inverse, $F_{k}$ are the components of the applied torques, $\tau^{a}$ are the magnitudes of the applied torques, and summation over repeated indices is implied. The Christoffel symbols are given by the usual formula

$$
\Gamma_{i j}^{k}=\frac{1}{2} \mathbb{G}^{k l}\left(\frac{\partial \mathbb{G}_{i l}}{\partial \theta_{j}}+\frac{\partial \mathbb{G}_{j l}}{\partial \theta_{i}}-\frac{\partial \mathbb{G}_{i j}}{\partial \theta_{l}}\right) .
$$

Because the $\ddot{\theta}$ terms are isolated, Equation 1 is only one step away from state space form and is a particularly convenient representation of the equations of motion. Define the input vector fields in the usual way by

$$
Y_{a}^{j}=\mathbb{G}^{j k} F_{k}^{a} \text {. }
$$

The $\mathbb{G}$-orthogonal complement to the input vector fields, denoted by $Y_{\perp}$, may be defined as a linearly independent set of unit-normal vector field that satisfies

$$
Y_{\perp}^{i} \mathbb{G}_{i j} \mathbb{G}^{j k} F_{k}^{a}=\left\langle\left\langle Y_{\perp}, \mathbb{G} Y_{a}\right\rangle\right\rangle=Y_{\perp}^{k} F_{a}^{k}=0
$$

and has unit length with respect to $\mathbb{G}$, i.e., $Y_{\perp}^{i} \mathbb{G}_{i j} Y_{\perp}^{j}=1$. For clarity of presentation, for the rest of this paper we will assume that the systems is underactuated by one input; however, we emphasize that the results are general and hold for any degree of underactuation.

The foundation of the approach is that a given velocity $v$ can be decomposed in the direction of the input vector fields and the orthogonal complement vector fields. In the case of a system like the three link manipulator, this will be of the form

$$
v=w_{1} Y_{1}+w_{2} Y_{2}+s Y_{\perp} .
$$

Since the $w_{1}$ and $w_{2}$ terms are the coefficients of $Y_{1}$ and $Y_{2}$, their rate of change is directly controlled by the inputs. In contrast, $s$ is the coefficient of the vector field orthogonal to them and can only be affected by the inputs through the coupling of the natural dynamics of the system. This decomposition requires that the input vector fields be linearly independent. If they are not, it may be assumed that a linearly independent subset is used. If Equation 2 is multiplied on the left by $\mathbb{G}$ and then $Y_{\perp}$, an expression for $s$ is obtained.

Recall that $Y_{1}$ and $Y_{2}$ are the input vector fields, so the $w_{1-}$ and $w_{2}$-components of the velocity are directly controlled. A straight-forward, but detailed, computation gives an expression for the derivative of $s$. In intrinsic form, it is given by

$$
\begin{aligned}
\frac{d}{d t} s(t)= & -w_{a}(t) w_{p}(t)\left\langle\left\langle\nabla_{Y_{a}} Y_{p}, Y_{\perp}\right\rangle\right\rangle \\
& -w_{a}(t) s(t)\left\langle\left\langle\nabla_{Y_{a}} Y_{\perp}, Y_{\perp}\right\rangle\right\rangle \\
& -s(t) w_{p}(t)\left\langle\left\langle\nabla_{Y_{\perp}} Y_{p}, Y_{\perp}\right\rangle\right\rangle \\
& -s(t) s(t)\left\langle\left\langle\nabla_{Y_{\perp}} Y_{\perp}, Y_{\perp}\right\rangle\right\rangle \\
& -\left\langle\left\langle\operatorname{grad} V, Y_{\perp}\right\rangle\right\rangle
\end{aligned}
$$

where $\langle\langle\rangle$,$\rangle is the inner product with respect to the inertia$ tensor and summation over repeated indices is implied. A complete derivation can be found in [10-12].

The critical point with respect to Equation 3 is that the inputs do not directly affect it. This is notationally clear simply from the fact that there are no inputs $\tau$ in the equation. It is only through the coupling of the dynamics with the directly controlled directions (the $w$ velocity directions), or the natural dynamics of the system, that $s$ changes. Hence, one measure of the control authority of the system is the magnitude of the coupling between the controlled velocities and $s$, which are the inner product terms on the first three lines of the equation. In coordinates, this expression is

$$
\begin{aligned}
\frac{d}{d t} s(t)= & -w_{a}(t) w_{p}(t)\left(\frac{\partial Y_{p}^{k}}{\partial \theta^{i}} Y_{a}^{i}+\Gamma_{i j}^{k} Y_{a}^{i} Y_{p}^{j}\right) \mathbb{G}_{k l} Y_{\perp}^{l} \\
& -s(t) w_{a}(t)\left(\frac{\partial Y_{\perp}^{k}}{\partial \theta^{i}} Y_{a}^{i}+\Gamma_{i j}^{k} Y_{a}^{i} Y_{\perp}^{j}\right) \mathbb{G}_{k l} Y_{\perp}^{l} \\
& -s(t) w_{p}(t)\left(\frac{\partial Y_{p}^{k}}{\partial \theta^{i}} Y_{\perp}^{i}+\Gamma_{i j}^{k} Y_{\perp}^{i} Y_{p}^{j}\right) \mathbb{G}_{k l} Y_{\perp}^{l} \\
& -s^{2}(t)\left(\frac{\partial Y_{\perp}^{k}}{\partial \theta^{i}} Y_{\perp}^{i}+\Gamma_{i j}^{k} Y_{\perp}^{i} Y_{\perp}^{j}\right) \mathbb{G}_{k l} Y_{\perp}^{l}-\frac{\partial V}{\partial \theta^{l}} Y_{\perp}^{l} .
\end{aligned}
$$

In Equations 3 and 4, the terms multiplying the $w$ and $s$ terms are what couple the dynamics between the directly controlled 
velocity directions and the velocity component orthogonal to them. Hence, the relevant terms that provide a measure of the dynamic coupling in the system are the three forms

$$
\begin{aligned}
B_{1}^{a p}\left(Y_{a}, Y_{p}\right) & =\left(\frac{\partial Y_{p}^{k}}{\partial \theta^{i}} Y_{a}^{i}+\Gamma_{i j}^{k} Y_{a}^{i} Y_{p}^{j}\right) \mathbb{G}_{k l} Y_{\perp}^{l} \\
B_{2}^{a}\left(Y_{a}\right) & =\left(\frac{\partial Y_{\perp}^{k}}{\partial \theta^{i}} Y_{a}^{i}+\Gamma_{i j}^{k} Y_{a}^{i} Y_{\perp}^{j}\right) \mathbb{G}_{k l} Y_{\perp}^{l} \\
B_{3}^{p}\left(Y_{p}\right) & =\left(\frac{\partial Y_{p}^{k}}{\partial \theta^{i}} Y_{\perp}^{i}+\Gamma_{i j}^{k} Y_{\perp}^{i} Y_{p}^{j}\right) \mathbb{G}_{k l} Y_{\perp}^{l} .
\end{aligned}
$$

It is these terms, when evaluated for all configurations along a trajectory, that provide a measure of the robustness of the control authority to disturbances in the uncontrolled velocity directions. These equations are presented for the case where the system is underactuated by one degree of freedom. Each additional degree of underactuation will add one additional set of these equations. For example, for a system underactuated by two, there will be six forms to consider. The term multiplying $s^{2}$ does not depend on either of the controlled velocities. Hence, it affects the rate of change of $s$, but does not provide any measure of how the control inputs can affect $s$. Note that these terms are directly computable and can be determined for any system for which the Lagrangian dynamics can be formulated.

\section{EXAMPlE: ThreE LiNK MANiPUlator}

As a specific example, consider the three-link manipulator (triple pendulum) in Figure 1 subjected to gravity with the base joint free and the other two actuated. The actuator at joint 2 is attached to link 2, so a positive actuator torque $\tau_{1}$ applies a positive torque to link 2 and a negative torque to link 1. Similarly, a positive actuator torque $\tau_{2}$ at joint 3 imparts a positive torque on link 3 and a negative torque on link 2. The system configuration is parametrized by the angles $\theta_{1}, \theta_{2}$ and $\theta_{3}$. The notation for the physical parameters is indicated in the figure.

The inertia tensor is given by

$$
\mathbb{G}=\left[\begin{array}{cc}
\frac{1}{4}\left(m_{1}+4\left(m_{2}+9 m_{3}\right)\right) l_{1}^{2}+J_{1} & \frac{1}{4} l_{1} l_{2}\left(m_{2}+6 m_{3}\right) \cos \theta_{12} \\
\frac{1}{4} l_{1} l_{2}\left(m_{2}+6 m_{3}\right) \cos \theta_{12} & \frac{1}{4}\left(m_{2}+4 m_{3}\right) l_{2}^{2}+J_{2} \\
\frac{3}{4} l_{1} l_{3} m_{3} \cos \theta_{13} & \frac{1}{4} l_{2} l_{3} m_{3} \cos \theta_{23} \\
\frac{3}{4} l_{1} l_{3} m_{3} \cos \theta_{13} \\
\frac{1}{4} l_{2} l_{3} m_{3} \cos \theta_{23} \\
\frac{m_{3} l_{3}^{2}}{4}+J_{3}
\end{array}\right],
$$

where $\theta_{i j}=\theta_{i}-\theta_{j}$. Using this metric, the kinetic energy is $T=\frac{1}{2} \dot{\theta}^{T} \mathbb{G} \dot{\theta}$, where $\dot{\theta}=\left[\begin{array}{lll}\dot{\theta}_{1} & \dot{\theta}_{2} & \dot{\theta}_{3}\end{array}\right]^{T}$. Since the system is subjected to gravity, we must consider the potential energy which is given by

$$
\begin{aligned}
V=\frac{1}{2} g\left(l _ { 1 } \left(m_{1}+\right.\right. & \left.2 m_{2}+6 m_{3}\right) \sin \theta_{1} \\
& \left.+l_{2}\left(m_{2}+2 m_{3}\right) \sin \theta_{2}+l_{3} m_{3} \sin \theta_{3}\right) .
\end{aligned}
$$

For $L=T-V$, the equations of motion are given by Lagrange's equations

$$
\frac{d}{d t}\left(\frac{\partial L}{\partial \dot{\theta}_{i}}\right)-\frac{\partial L}{\partial \theta_{i}}=F_{i}^{1}+F_{j}^{2}
$$

where

$$
F^{1}=\tau_{1}\left[\begin{array}{c}
-1 \\
1 \\
0
\end{array}\right], \quad \text { and } \quad F^{2}=\tau_{2}\left[\begin{array}{c}
0 \\
-1 \\
1
\end{array}\right] .
$$

The idea is to consider velocity components aligned with and orthogonal to the inputs (orthogonal with respect to the kinetic energy metric). Thus, a velocity is expressed in terms of the magnitude of three components, $w_{1}, w_{2}$ and $s$, where

$$
\begin{aligned}
v & =\left[\begin{array}{c}
\dot{\theta}_{1} \\
\dot{\theta}_{2} \\
\dot{\theta}_{3}
\end{array}\right] \\
& =w_{1} \mathbb{G}^{-1}\left[\begin{array}{c}
-1 \\
1 \\
0
\end{array}\right]+w_{2} \mathbb{G}^{-1}\left[\begin{array}{c}
0 \\
-1 \\
1
\end{array}\right]+s\left[\begin{array}{l}
1 \\
1 \\
1
\end{array}\right] .
\end{aligned}
$$

Note that $w_{1}$ and $w_{2}$ are directly aligned with the input torques and the $s$ component is orthogonal (specifically, $\mathbb{G}$ orthogonal) to both. The presence of the inverse of the inertia tensor before the first two vectors arises from converting Lagrange's equations to state space form, as detailed subsequently.

Since the first two terms are directly aligned with the inputs, they can be directly controlled. An expression for how $s$ changes with time provides a measure of the coupling between the controlled velocities, $w_{1}$ and $w_{2}$, and the orthogonal velocity $s$. Unsurprisingly, this coupling depends on the configuration of the robot. In some configurations, the dynamics are completely decoupled, indicating that there is no instantaneous control authority over the orthogonal direction. In other configurations, there is tight coupling.

The practical implication is that the expression for this coupling provides a measure of instantaneous (i.e., highbandwidth) control authority over the orthogonal direction, which may be interpreted as a measure of a type of dynamic mechanical advantage.

The following simulations show the dynamic response of the three-link manipulator in three configurations subjected to large inputs. In all cases, the acceleration of gravity $g=1$, and the parameters are

$$
\begin{array}{lll}
l_{1}=1 & m_{1}=4 & J_{1}=7 \\
l_{2}=2 & m_{2}=5 & J_{2}=8 \\
l_{3}=3 & m_{3}=6 & J_{3}=9 .
\end{array}
$$

These values were specifically selected to avoid any simplifications that may occur in more symmetric designs, and the intentionally omitted units are taken to be consistent. The three configurations considered are illustrated in Figure 2. For configuration 1,

$$
\left(\theta_{1}, \theta_{2}, \theta_{3}\right)=(0.25,3.74,2.84),
$$




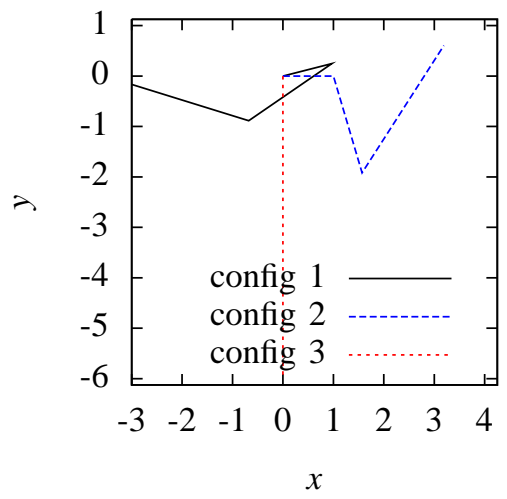

Fig. 2. The three configurations of the threelink manipulator.

which places the manipulator near a dynamic singularity, although this fact is not at all obvious from a simple inspection of the configuration. For configuration 2,

$$
\left(\theta_{1}, \theta_{2}, \theta_{3}\right)=(0,5,1)
$$

which places the manipulator away from a dynamic singularity. Finally, for configuration 3 ,

$$
\left(\theta_{1}, \theta_{2}, \theta_{3}\right)=\left(-\frac{\pi}{2},-\frac{\pi}{2},-\frac{\pi}{2}\right),
$$

which corresponds to both a kinematic and a dynamic singularity of the mechanism. Note that neither of the first two configurations is near a kinematic singularity.

To give a feel for the relative complexity of the equations, $Y_{1}, Y_{2}$ and $Y_{\perp}$ are given in vector form as

$$
\begin{aligned}
& Y_{1}= \\
& \frac{4\left(178 \cos \theta_{12}-27 \cos \left(\theta_{1}+\theta_{2}-2 \theta_{3}\right)-18 \cos 2 \theta_{23}+352\right)}{6191 \cos 2 \theta_{12}+378005 \theta_{13}+2610 \cos 2 \theta_{23}-82151} \\
& 6191 \cos 2 \theta_{12}+3780 \cos 2 \theta_{13}+2610 \cos 2 \theta_{23}-82151 \\
& 2\left(-356 \cos \theta_{12}+54 \cos \left(\theta_{1}+\theta_{2}-2 \theta_{3}\right)+81 \cos 2 \theta_{13}-1259\right) \\
& \begin{array}{l}
6191 \cos 2 \theta_{12}+3780 \cos 2 \theta_{13}+2610 \cos 2 \theta_{23}-82151 \\
2\left(-362 \cos \theta_{13}-123 \cos \left(2 \theta_{12}-\theta_{3}\right)+413 \cos \theta_{23}+82 \cos \left(\theta_{1}-2 \theta_{2}+\theta_{3}\right)\right) \\
\hline
\end{array} \\
& Y_{2}= \\
& \frac{6191 \cos 2 \theta_{12}+3780 \cos 2 \theta_{13}+2610 \cos 2 \theta_{23}-82151}{\left.\cos \theta_{13}-123 \cos \left(2 \theta_{12}-\theta_{3}\right)+413 \cos \theta_{23}+82 \cos \left(\theta_{1}-2 \theta_{2}\right)\right)} \\
& {\left[\begin{array}{l}
- \\
2 \\
2
\end{array}\right.} \\
& -\frac{4\left(178 \cos \theta_{12}-27 \cos \left(\theta_{1}+\theta_{2}-2 \theta_{3}\right)-181 \cos \theta_{13}+41 \cos \left(\theta_{1}-2 \theta_{2}+\theta_{3}\right)\right)}{6191 \cos 2 \theta_{1}+3780 \cos 2 \theta_{13}+2610 \cos 2 \theta_{23}-82151} \\
& \begin{array}{r}
6191 \cos 2 \theta_{12}+3780 \cos 2 \theta_{13}+2610 \cos 2 \theta_{23} \\
2\left(-81 \cos 2 \theta_{13}-123 \cos \left(2 \theta_{12}-\theta_{3}\right)+413 \cos \theta_{23}+1259\right) \\
\hline 6191 \cos 2 \theta_{12}+3780 \cos 2 \theta_{1}+2610 \cos 2 \theta_{23}-82151
\end{array} \\
& 6191 \cos 2 \theta_{12}+3780 \cos 2 \theta_{13}+2610 \cos 2 \theta_{23}-82151 \\
& \frac{2\left(1681 \cos 2 \theta_{12}+1107 \cos \left(2 \theta_{12}-\theta_{3}\right)-7\left(531 \cos \theta_{23}+2593\right)\right)}{9\left(6191 \cos 2 \theta_{12}+3780 \cos 2 \theta_{13}+2610 \cos 2 \theta_{23}-82151\right)} \\
& Y_{\perp}=\left[\begin{array}{c}
\frac{2}{\sqrt{164 \cos \theta_{12}+108 \cos \theta_{13}+72 \cos \theta_{23}+506}} \\
\frac{2}{\sqrt{164 \cos \theta_{12}+108 \cos \theta_{13}+72 \cos \theta_{23}+506}} \\
\frac{2}{\sqrt{164 \cos \theta_{12}+108 \cos \theta_{13}+72 \cos \theta_{23}+506}}
\end{array}\right] \text {. }
\end{aligned}
$$

Observe that $Y_{\perp}$ is the same form as in Equation 7 except for a configuration-dependent scaling.

The response of the system is computed for nine cases, three for each of the three configurations:

1) no input torques;

2) case 1: $\tau_{1}=1000$ and $\tau_{2}=-1000$; and,

3) case 2: $\tau_{1}=-1000$ and $\tau_{2}=1000$.

The velocities for the initial conditions in the simulations were chosen to give the same non-zero magnitude of the uncontrolled velocity direction. While the degree of coupling

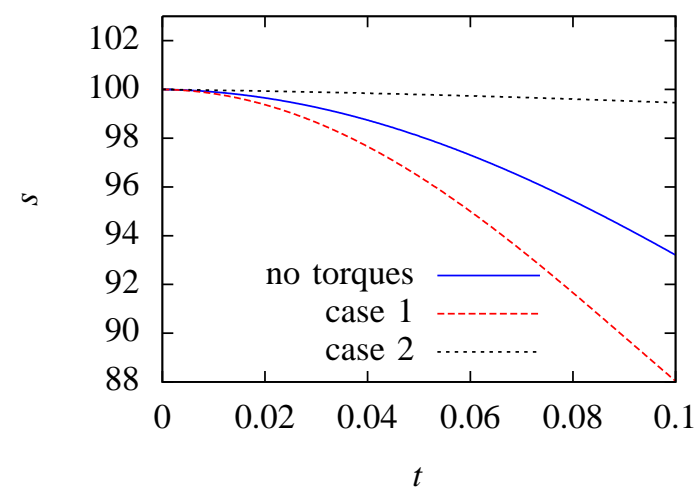

Fig. 3. Good control authority illustrated in the response away from a dynamic singularity in configuration 2 .

is a function of the configuration and system parameters only, the coupling itself is proportional to the velocities, so a non-zero velocity is necessary for there to be any coupling. Furthermore, nonzero velocities are more representative of a robotic manipulator or system performing some task.

The responses of the uncontrolled velocity direction for the non-singular configuration 2 are illustrated in Figure 3, and for the dynamically singular configuration 1 in Figure 4 and the dynamically singular case 3 in Figure 5. Relatively large torques and a short time interval were chosen to be representative of the dynamics and characteristic of a manipulator performing a dynamically demanding task. Note that away from a dynamic singularity (the right-hand plot in Figure 2), there is significant control authority in that the application of the inputs significantly alters the magnitude of the uncontrolled velocity. In contrast, for the configurations near the dynamic singularities illustrated in Figures 4 and 5, there is only a very limited response.

The proper interpretation of the responses is the difference between the "no torques" curves and the two numbered cases. Where there is a significant difference with and without applied torques, significant coupling exists between the controlled and uncontrolled directions. Where there is little difference, the responses are illustrative of a dynamic singularity. The fact that there is any difference at all in the singular cases is due to the fact that the system has nonzero initial velocity and leaves the singularity. Therefore, in Figure 3, the large difference between the middle and outer curves is a manifestation of strong coupling and hence good control authority. The small differences between the three curves in the plots in Figures 4 and 5 is a manifestation of the dynamic singularity and hence poor control authority.

The expression for the coupling forms in Equation 5 are too long to present with the parameter values unspecified. For the same numerical parameter values as in the simulations, direct computations give expressions for these coupling terms. In particular, the first form $B_{1}$, which is the 


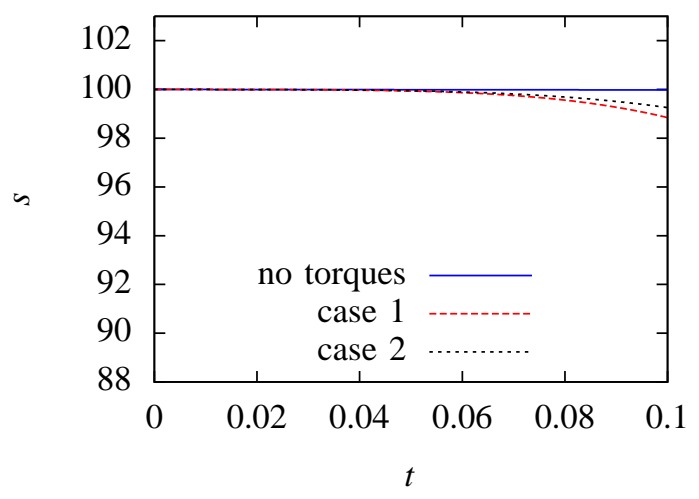

Fig. 4. Response near configuration 1, a dynamic singularity, illustrating a weak control response.

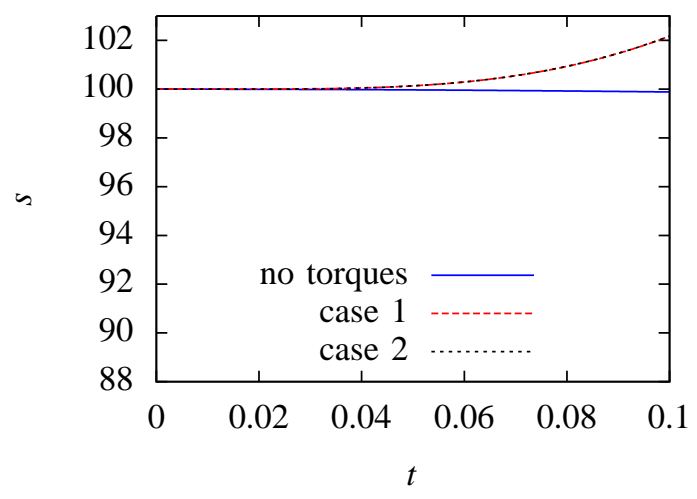

Fig. 5. Response near configuration 3, which is at both a dynamic and a kinematic singularity (the responses for cases 1 and 2 are the same for this configuration), illustrating a weak response.

term multiplying $w_{a}(t) w_{p}(t)$, is identically zero. The second and third forms are not zero and are relatively complicated. The numerator of the second form $B_{2}$, the term multiplying $s(t) w_{1}(t)$, is

$$
\begin{aligned}
& -2\left(69251 \sin \left(\theta_{12}\right)+12382 \sin \left(2\left(\theta_{12}\right)\right)+\right. \\
& 9\left(-155 \sin \left(\theta_{1}+\theta_{2}-2 \theta_{3}\right)+1702 \sin \left(\theta_{13}\right)+\right. \\
& 420 \sin \left(2\left(\theta_{13}\right)\right)+453 \sin \left(2 \theta_{12}-\theta_{3}\right)-1903 \sin \left(\theta_{23}\right)- \\
& \left.\left.290 \sin \left(2\left(\theta_{23}\right)\right)+302 \sin \left(\theta_{1}-2 \theta_{2}+\theta_{3}\right)\right)\right),
\end{aligned}
$$

and the denominator is

$$
\begin{aligned}
\left(82 \cos \left(\theta_{12}\right)+54 \cos \left(\theta_{13}\right)+\right. & \left.36 \cos \left(\theta_{23}\right)+253\right) \\
\left(6191 \cos \left(2\left(\theta_{12}\right)\right)+\right. & 3780 \cos \left(2\left(\theta_{13}\right)\right)+ \\
& \left.2610 \cos \left(2\left(\theta_{23}\right)\right)-82151\right) .
\end{aligned}
$$

The numerator of the third form $B_{3}$, the term multiplying $s(t) w_{p}(t)$, is

$$
\begin{gathered}
-2\left(-44383 \sin \left(\theta_{12}\right)-6191 \sin \left(2\left(\theta_{12}\right)\right)+\right. \\
3915 \sin \left(\theta_{1}+\theta_{2}-2 \theta_{3}\right)+43465 \sin \left(\theta_{13}\right)+ \\
3780 \sin \left(2\left(\theta_{13}\right)\right)+1663 \sin \left(2 \theta_{12}-\theta_{3}\right)+ \\
44327 \sin \left(\theta_{23}\right)+5220 \sin \left(2\left(\theta_{23}\right)\right)- \\
\left.5945 \sin \left(\theta_{1}-2 \theta_{2}+\theta_{3}\right)\right),
\end{gathered}
$$

and the denominator is the same. When these coupling terms are zero, the system is at a dynamic singularity because the orthogonal velocity component is not affected at all by the controlled velocity directions. Hence, from a disturbance rejection perspective, it is desirable for the coupling terms to be large.

While complicated, it is relatively simple to find configurations where these coupling terms are zero. The angles of singular configuration 1 in the simulation example, $\left(\theta_{1}, \theta_{2}, \theta_{3}\right)=(0.25,3.74,2.84)$, were readily computed numerically. More intuitively, by inspection of the numerator terms, it is clear that they are zero when all the angles have the same value. The consequence, again, is that if these terms are zero even with arbitrarily large torques, the $s$ velocity cannot be altered until the mechanism moves away from the configuration. In contrast, away from such points, the control authority is much better. Such configurations with large coupling will exist when the denominator term is small and the numerators are large.

\section{CONCLUSIONS AND Future WORK}

This paper presents a measure of dynamic singularities for mechanical robotic systems and demonstrated with a realistically-complicated example the effect and nature of dynamic singularities for such systems. They are clearly problematic for underactuated systems because dynamic coupling is necessary for complete control authority, and in some configurations this dynamic coupling will be zero. Simulation results demonstrated that, analogous to kinematic singularities, near a dynamic singularity even with very large input torques there is very little effect on the underactuated velocity directions. The measure is very general in that it applies to any underactuated mechanical system that is governed by Lagrange's equations where the Lagrangian is kinetic minus potential energy.

There are three areas of future work we intend to pursue.

1) The analysis of dynamic singularities should be very useful in feedback control design for disturbance rejection. Analogous to the notion of the controllability index for linear systems (see [3]) the expressions for the forms given in Equation 5 provides a measure of control authority over the unactuated degrees of freedom. In the case where some of the disturbances are likely to be in the uncontrolled directions, it will generally be desirable to operate the system away from the dynamic singularities. On the other hand, if the disturbances are generally aligned with the controlled 
directions, then operating near a dynamic singularity would be advantageous because the uncontrolled directions will actually be isolated from the disturbances due to the lack of dynamic coupling.

2) With a computable expression for the degree of coupling between the controlled and uncontrolled directions, mechanisms may be designed to minimize or eliminate dynamic singularities or to ensure they are in configurations where they are as inconsequential as possible. In the latter case, it may be desirable the singularities can be placed in a region of the configuration space where the mechanism is not intended to operate. Of course, this would make operating in the unintended configurations even more problematic, but that is a consideration the designer would have to consider.

3) The above two considerations will be applied to the specific problem of dynamic bipedal robotic locomotion. Specifically, when designing a gait for a robot, ensuring maximal coupling throughout the gait will provide additional robustness for the gait. This can be accomplished in two stages, first during the design of the robot and then secondly when designing baseline locomotion gaits for it.

\section{ACKNOWLEDGMENTS}

The authors gratefully acknowledge the support of the Center for Applied Mathematics at the University of Notre Dame.

\section{REFERENCES}

[1] R. Murray A. Lewis, J.P. Ostrowski and J. Burdick. Nonholonomic mechanics and locomotion: the snakeboard example. In Proceedings of the 1994 IEEE International Conference on Robotics and Automation, pages 2391-2400, 1994.

[2] Ralph Abraham and Jerrold E. Marsden. Foundations of Mechanics. Addison Wesley, second edition, 1978.

[3] Panos J. Antsaklis and Anthony N. Michel. Linear Systems. McGraw Hill, 1997.

[4] F. Bullo and A. D. Lewis. Geometric Control of Mechanical Systems, volume 49. Springer-Verlag, 2004.

[5] F. Bullo and M. Zefran. On mechanical control systems with nonholonomic constraints and symmetries,. Systems and Control Letters, 45(2):133-143, 2002.
[6] Francesco Bullo and Andrew D. Lewis. Kinematic controllability and motion planning for the snakeboard. IEEE Transactions on Robotics and Automation, 19(3):494-498, 2003.

[7] Francesco Bullo and Andrew D. Lewis. Low-order controllablility and kinematic reductions for affine connection control systems. SIAM Journal on Control and Optimization, 44(3):885-908, 2005.

[8] S. Chitta and V.J. Kumar. Dynamics and generation of gaits for a planar rollerblader. In Proceedings of the 2003 IEEE/RSJ International Conference on Intelligent Robots and Systems, pages 860-865, 2003.

[9] A. Lewis F. Bullo, J. Cortes and S. Martinez. Vectorvalued quadratic forms in control theory. In V. Blondel and A. Megretski, editors, Sixty Open Problems in Mathematical Systems and Control Theory, pages 315320. Princeton University Press, 2004.

[10] Jason Nightingale, Richard Hind, and Bill Goodwine. Geometric analysis of a class of constrained mechanical control systems in the nonzero velocity setting. In Proceedings of the 2008 International Federation of Automatic Control World Congress.

[11] Jason Nightingale, Richard Hind, and Bill Goodwine. A stopping algorithm for mechanical systems underactuated by one control. In Proceedings of the 2008 Workshop on the Algorithmic Foundations of Robotics (WAFR).

[12] Jason Nightingale, Richard Hind, and Bill Goodwine. Intrinsic vector-valued symmetric form for simple mechanical control systems in the nonzero velocity setting. In Proceedings of the 2008 International Conference on Robotics and Automation, 2008.

[13] J.P. Ostrowski, J.P. Desai, and V. Kumar. Optimal gait selection for nonholonomic locomotion systems. The International Journal of Robotics Research, 19(5)::225-237, 2000.

[14] Evangelos Papadopoulos and Steven Dubowsky. Dynamic singularities in free-floating space manipulators. Journal of dynamic systems, measurement, and contro, 115(1):44-52, 1993.

[15] E.R. Westervelt, B. Morris, and K.D. Farrell. Analysis results and tools for the control of planar bipedal gaits using hybrid zero dynamics. Auton Robot, 23(2):131145, 2007. 